\title{
PERFIL DOS IDOSOS ATENDIDOS EM UM CENTRO DE ATENÇÃO PSICOSSOCIAL - ÁLCOOL E OUTRAS DROGAS
}

\author{
Profile of the elderly assisted in a psychosocial care center - alcohol and other drugs \\ Perfil de los ancianos asistido en el centro de atención psicosocial - alcohol y otras \\ droga
}

Sandra Cristina Pillon ${ }^{1}$

Lucilene Cardoso²

Gisela Amorim Marques Pereira ${ }^{3}$

Emmanuel Mello ${ }^{4}$

\section{RESUMO}

0 objetivo do estudo foi identificar o perfil dos idosos usuários de substâncias psicoativas atendidos no Centro de Atenção Psicossocial de álcool e drogas no interior paulista, no período de 1996 a 2009. 0 desenho metodológico é descritivo, do tipo retrospectivo, baseado em dados secundários. A amostra foi composta por 191 clientes com idade acima de 60 anos, que correspondem a $3,2 \%$ de todos os atendidos no referido serviço. Os idosos caracterizaram-se por serem predominantemente do sexo masculino; a média de idade foi 64 anos, com baixo nível de escolaridade; 78,4\% tinham até o ensino fundamental; e 86\% eram aposentados. As drogas de maior uso foram: o álcool, a maconha, o cracke a cocaína. Conclui-se que o número de idosos que buscam assistência especializada é muito baixo. Estudos dessa natureza são importantes para avaliar as necessidades desse grupo em evidente crescimento, bem como o uso de substâncias psicoativas para o planejamento de práticas efetivas.

Palavras-chave: Idoso. Transtornos Relacionados ao Uso de Substâncias. Centros de Tratamento de Abuso de Substâncias.

\begin{abstract}
The objective was to identify the profile of the elderly at the Center for Psychosocial Care - alcohol and other drugs in Ribeirão Preto SP, in the period of 1996 to 2009. The methodological design was the descriptive retrospective study based on secondary data. The sample consisted of 191 (3.2\%) customer over 60 years assisted in the service. The sample was characterized by elders with a mean age of 64 years, predominantly male, with low educational level, $78.4 \%$ had primary school and $86 \%$ were retired. The most commonly drugs used were alcohol, marijuana, crack and cocaine. It concludes that the number of elderly who seeks for expert assistance is very low. Studies of this nature are important to assess the needs of this group in the clear growth as well as the use of psychoactive substances for planning effective practices.
\end{abstract}

Key-words: Aged. Substance-related disorders. Substance abuse treatment centers

\section{Resumen}

El objetivo del estudio fue identificar el perfil de los ancianos del Centro de Atención Psicosocial de alcohol y otras drogas de Ribeirao Preto, SP, en el período 1996 a 2009. El diseño metodológico es descriptivo del tipo retrospectivo basado en los datos secundarios. La muestra consistió de 191 (3,2\%) clientes mayores de 60 años asistidos en ese servicio. La muestra se caracterizó por los ancianos con media de edad de 64 años con un predominio masculino, con bajo nivel educacional: $78,4 \%$ tenía estudios primarios y el $86 \%$ eran jubilados. Las drogas de mayor uso fueron: alcohol, marihuana, crack y cocaína. Se concluye que el número de ancianos que buscan la asistencia de expertos es muy bajo. Estudios de esa naturaleza son importantes para evaluar las necesidades de ese grupo en el evidente crecimiento, así como el uso de sustancias psicoactivas para la planificación de prácticas efectivas.

Palabras-clave: Anciano. Trastornos relacionados con sustancias. Centros de tratamiento de abuso de sustancias.

'Professor Associado. Departamento de Enfermagem Psiquiátrica e Ciências Humanas da Universidade de São Paulo - EERP-USP. Líder do Grupo de Estudo sobre Prevenção do Uso Nocivo do Álcool e ou Drogas - GRUPAD (CNPq/USP). Ribeirão Preto - SP. Brasil. E-mail: pillon@eerp.usp.br ,2Professor Doutor. Departamento de Enfermagem Psiquiátrica e Ciências Humanas. Escola de Enfermagem de Ribeirão Preto. Universidade de São Paulo. Ribeirão Preto - SP. Brasil. E-mail: cardoso@eerp.usp.br, ${ }^{3}$ Terapeuta Ocupacional. Mestre em Ciências pelo Programa de Pós-Graduação em Enfermagem Psiquiátrica da Escola de Enfermagem de Ribeirão Preto. Profissional do Centro de Atenção Psicossocial - Álcool e Drogas de Ribeirão Preto-SP. Brasil. E-mail: giamorim@uol.com.br, ${ }^{4}$ Psicólogo. Mestre em Filosofia pelo Programa de Pós-graduação em Filosofia do Departamento de Filosofia e Metodologia Científica da Universidade Federal de São Carlos. Profissional do Centro de Atenção Psicossocial - Álcool e Drogas de Ribeirão Preto-SP. Brasil. E-mail: emmanuelmello@yahoo.com.br 


\section{INTRODUCÃO}

0 relatório sobre os indicadores sociodemográficos e de saúde no Brasil demonstrou que em 2003, de acordo a Pesquisa Nacional por Amostra de Domicílios (PNAD), a população idosa (acima de 60 anos) era de aproximadamente $17(10 \%)$ milhões. ${ }^{1}$ Anos mais tarde, esse órgão demonstrou que o número de idosos aumentou significativamente, para 19 milhões de pessoas, evidenciando o acelerado processo de envelhecimento da sociedade brasileira. ${ }^{1}$ Estima-se que ,até 0 ano de 2025, o Brasil será o sexto país do mundo com maior número de pessoas idosas. ${ }^{1}$ Este fenômeno se deve especialmente ao incremento na expectativa de vida, de forma que grande parte da população atual irá alcançar a velhice, tornando-se um grande desafio para os próximos anos.

Diante do crescimento de pessoas idosas, um problema preocupante para os profissionais de saúde e a Saúde Pública tem sido o uso de substâncias psicoativas. Embora a literatura aponte que a prevalência do uso de álcool tem sido maior entre os jovens e que diminui com a maturidade, o número de usuários de substâncias psicoativas continuará a aumentar com 0 crescimento da proporção de idosos. ${ }^{2}$ No entanto, a prevalência de uso de álcool em idosos geralmente é mais aceita por ser menor que a dos jovens, mas, infelizmente, ainda pouco se sabe sobre o abuso e a dependência das substâncias psicoativas nesse grupo da população.

0 uso de álcool entre idosos foi descrito como um problema complexo, multifatorial, um fenômeno não muito bem entendido, ${ }^{3}$ caracterizado por uma epidemia invisível, uma vez que os problemas e, por conseguinte, os índices são subestimados e mal identificados. ${ }^{4}$ Sobre essa temática, existe uma notável limitação na literatura científica brasileira e a maioria dos estudos é internacional.

Dada esta condição, o presente estudo tem por objetivo identificar o perfil dos idosos atendidos em um Centro de Atenção Psicossocial de álcool e drogas do interior paulista.

\section{MARCO TEÓRICO}

A literatura evidencia que a carência de estudos sobre o tema do uso de substâncias psicoativas entre idosos pode estar relacionada às dificuldades de identificação do uso das substâncias (padrão de consumo, tipo de substância usada, níveis de gravidade da dependência) e os problemas (físicos, psicológicos e sociais), tanto por parte do próprio idoso, ou mesmo pelos profissionais de saúde. ${ }^{3,4}$

Quanto aos idosos, por diversos motivos (vergonha, medo, demência, estilo de vida, isolamento), não relatam seu consumo, o que dificulta ainda mais a identificação e posterga o início de uma intervenção precoce. Já em relação aos profissionais de saúde, podem estar relutando em investigar tal consumo e suas consequências, seja pela falta de habilidades técnicas, ou mesmo pela imagem estereotipada de que o uso do álcool atinge apenas os jovens.

Um terceiro fator centra-se na dificuldade em identificar os sinais e sintomas do uso por estes estarem também relacionados à suas consequências e por si não serem tão específicos, tais como acidentes, transtornos mentais (depressão, ansiedade, demência) insônia e autonegligência, sintomas que podem estar mistificados aos do processo de envelhecimento. ${ }^{5}$

A prevalência do uso, abuso e dependência de substância psicoativa entre os idosos varia muito, pois depende do método utilizado para detectar o uso e as consequências, a distribuição geográfica e a amostra (geralmente são utilizadas sub amostras), o que dificulta uma comparação. ${ }^{6} \mathrm{~A}$ literatura evidenciou que, entre os idosos, os fatores sociodemográficos mais comuns associados ao consumo de álcool envolvem o sexo masculino, o isolamento social e o estado civil (solteiro, separado ou divorciado). ${ }^{?}$

Dentre os fatores associados ao uso de drogas, a literatura enfatizou que, embora com a maturidade 0 adulto tenha menos probabilidade de usar alguma droga, uma vez exposto, é particularmente vulnerável ao desenvolvimento da dependência., ${ }^{5,-8} 0$ aumento dos riscos são resultados de vários fatores relacionados à idade, incluindo o aumento da fragilidade, mudanças corpóreas e no metabolismo das drogas, aumento da morbidade e da utilização de medicações prescritas (incluindo as medicações de abuso com risco potencial para 0 desenvolvimento da dependência). ${ }^{5-8}$

Em relação aos estudos epidemiológicos, embora desenvolvidos com diferentes metodologias e locais, estima-se de $2 \%$ a $20 \% \%^{8-9}$ os idosos que fazem uso abusivo de álcool, pois esta é droga de maior consumo nessa faixa etária. Estimase que $1 \%$ a $12 \%^{3}$ apresentem critérios de diagnóstico da síndrome da dependência, e, outros estudos mencionaram que dependendo do ambiente de tratamento, esses índices são ainda maiores: $14 \%$ nas urgências hospitalares, $18 \%$ a $41 \%$ nas internações em enfermarias e $23 \%$ a $44 \%$ em enfermarias psiquiátricas. ${ }^{10-11}$

Um estudo americano divulgou que um terço da população idosa (acima de 60 anos) consumiu álcool e 3,5\% consumiram uma ou mais substâncias ilícitas no último ano (idade maior que 50 anos). ${ }^{12} 0$ uso e o abuso de álcool e ou de substâncias psicoativas por idosos provocam efeitos claros e profundos na saúde e no bem-estar dos idosos em todos os aspectos da vida e são potenciais de risco para o desenvolvimento de problemas físicos, psicológicos (autoestima, habilidade de enfrentamento, comprometimento das relaç̃̃es interpessoais) e sociais, muitas vezes detectados nos diversos níveis de atenção de cuidados à saúde. ${ }^{2}$

As estimativas da prevalência atual de álcool e ou de drogas na população idosa variam, mas os resultados desse consumo, quando associados ao processo de envelhecimento, geram impactos nos cuidados de saúde e altos custos sociais. $^{12}$ 
0 consumo de substâncias psicoativas está associado a altos índices de morbidade e mortalidade entre os idosos. Dessa forma, torna-se imperativo que todos os profissionais de saúde tenham conhecimentos técnicos específicos sobre o uso abusivo e a dependência de álcool e/ou drogas na população idosa, para que possam oferecer uma assistência digna e de qualidade. ${ }^{13}$

Porém, existem poucas informações atuais disponíveis a respeito do uso de álcool em idosos, principalmente nos serviços especializados em tratamento de substâncias psicoativas. No Brasil, os Centros de Atenção Psicossocial de álcool e outras drogas (CAPS-ad) em cidades com mais de 70 mil habitantes foram abertos para atender a demanda de usuários de álcool e de outras drogas independente da idade; no entanto, pouco se sabe a respeito de quem são os idosos atendidos neste serviço, fato que justifica a realização do presente estudo.

0 objetivo do presente estudo foi identificar o perfil dos idosos atendidos em um Centro de Atenção Psicossocial de álcool e outras drogas do interior paulista.

\section{METODOLOGIA}

0 desenho metodológico do estudo é do tipo descritivo retrospectivo baseado em dados secundários, em que se avaliou o período de 1996 a 2009 . 0 estudo faz parte de um estudo maior dos pesquisadores, subvencionado pelo Conselho Nacional de Desenvolvimento Científico e Tecnológico (CNPq). 0 estudo foi realizado em um serviço especializado no tratamento da dependência de álcool e outras drogas, o Centro de Atenção Psicossocial Álcool e Drogas de Ribeirão Preto, SP. Para compor a amostra realizou-se uma avaliação no banco de dados do presente serviço; em seus 13 anos de assistência, identificaram-se 5.889 (100\%) clientes registrados no serviço. Foram incluídos apenas os clientes com idade acima de 60 anos, ou seja, 191 (3,2\%) idosos atendidos no presente serviço. Para a coleta de dados elaborou-se um questionário contendo as informações sociodemográficas e o tipo de drogas utilizadas.

Quanto aos aspectos éticos, o presente estudo foi submetido e aprovado (Processo N. 0705/2006) pelo Comitê de Ética em Pesquisa da Escola de Enfermagem da Universidade de São Paulo, seguindo a Resolução 196/96 CONEP. Um banco de dados foi elaborado no Statistical Package Social Science (SPSS) na versão Windows para a inserção e análise dos dados. Os resultados foram apresentados em números e porcentagens.

\section{RESULTADOS}

Dos 5.889 prontuários cadastrados no serviço desde sua abertura, 191 (3,3\%) eram de pacientes com idade acima dos 60 anos.

Dentre as informações sociodemográficas, a média de idade foi de 64 anos (DP $\pm 4,62$ ), com variação de 60 a 81 anos; houve predomínio do sexo masculino, 173 (90,6\%), e baixo nível de escolaridade, $150(78,4 \%)$ tinham até o ensino fundamental e $86(45 \%)$ eram aposentados, conforme apresentado na Tabela 1.

Tabela 1. Informações sociodemográficas dos clientes idosos do CAPS-ad ( $N=191$ ).

\begin{tabular}{llcc}
\hline & & $\mathbf{N}$ & $\mathbf{\%}$ \\
\hline Sexo & Masculino & 173 & 90,6 \\
& Feminino & 18 & 9,4 \\
& Analfabetos & 24 & 12,6 \\
& Ensino Fundamental & 150 & 78,5 \\
& Ensino Médio & 8 & 4,2 \\
Ocupação & Ensino Superior & 9 & 4,7 \\
& Aposentados & 86 & 45,0 \\
& Desempregados & 65 & 34,0 \\
& Trabalham & 38 & 19,9 \\
& Do lar & 2 & 1,0 \\
\hline
\end{tabular}


Tabela 2. Distribuição de frequência e porcentagem dos clientes idosos do CAPS-ad em relação ao tipo de drogas utilizadas ( $N=191)$.

\begin{tabular}{lcc}
\hline & N & $\%$ \\
\hline Álcool & 160 & 83,8 \\
Medicação de abuso & 5 & 2,6 \\
Álcool e Cocaína ou Crack & 5 & 2,6 \\
Maconha & 4 & 2,1 \\
Tabaco & 3 & 1,6 \\
Álcool, Cocaína, Crack e Maconha & 3 & 1,6 \\
Álcool e Crack & 2 & 1,0 \\
Álcool e Maconha & 2 & 1,0 \\
Álcool e Medicação & 2 & 1,0 \\
Cocaína ou Crack e Maconha & 2 & 1,0 \\
Álcool, Crack, Maconha e Medicação & 1 &, 5 \\
Álcool, Crack, Maconha e Solventes & 1 &, 5 \\
Não Respondeu & 1 &, 5 \\
\hline
\end{tabular}

Em relação ao uso de substâncias psicoativas, a droga mais consumida foi o álcool 160 (83,8\%), conforme demonstra a Tabela 2.

\section{DISCUSSÃO}

Dos clientes atendidos no serviço especializado em tratamento aos usuários de substâncias psicoativas, no período de 13 anos de assistência, 3,2\% eram idosos. Esses dados corroboram os apresentados na literatura ${ }^{2-8}$, uma vez que 0 número de pessoas nessa faixa etária que busca assistência de saúde em serviços especializados para o tratamento do uso de substâncias psicoativas tem sido muito baixo.

Por ser um estudo descritivo, vale enfatizar que, na literatura, não foram identificados estudos específicos sobre o uso de substâncias psicoativas em idosos clientes de CAPS-ad, principalmente no Brasil. Os estudos disponíveis sobre essa temática são epidemiológicos, desenvolvidos com diversas metodologias e amostras, e outros estudos são de revisão de literatura. Assim, os resultados discutidos no presente estudo enfocam os principais dados da literatura, no que refere às informações sociodemográficas e substâncias psicoativas consumidas pelos idosos, sem o intuito de comparar, mas de levantar os fatores que podem favorecer o uso, o abuso e a dependência de substâncias psicoativas, como meio de repensar a prática assistencial a esse grupo da população.
Referente aos resultados obtidos que delinearam 0 perfil dos poucos idosos atendidos no serviço $(3,2 \%)$, sendo estes predominantemente do sexo masculino, aposentados, com baixo nível de escolaridade, estudos reforçam a importância e necessidade de mais pesquisas nesta temática.

A literatura de consenso apontou que o maior consumo de qualquer substância psicoativa está muito mais presente entre os jovens, e que o padrão de consumo tende a ser bem diferente dos idosos e esse fato pode modificarse com a idade, ${ }^{5-13}$ o que, no entanto, não desmerece uma atenção especial nas demais faixas etárias, principalmente entre os idosos, por esta ser uma população que tem sido pouco estudada.

0 alcoolismo em jovem (60\%) é notavelmente mais identificado que em idosos (38\%), pois constatou-se que havia um menor número de encaminhamentos de idosos aos serviços especializados. E, ainda, porque esses apresentavam poucas chances de serem diagnosticados caso apresentassem características como ser branco, do sexo feminino ou tivessem bom nível de escolaridade. ${ }^{14}$

Em relação ao gênero, a literatura demonstrou que, geralmente, os homens idosos bebem mais que as mulheres idosas, e estas apresentam maiores probabilidades de modificarem o comportamento do beber com a idade 3 , fato que pode justificar o baixo número de mulheres no tratamento (menos que 10\%). 
No presente estudo, evidenciou-se que o consumo de bebidas alcoólicas ocorreu mais entre os homens; em relação aos demais grupos da população, isso pode estar relacionado ao fato de que, diante do adoecimento, eles apresentam melhor aceitação (social/cultural) e, assim, buscam tratamento.

Além disso, um estudo demonstrou que é duas vezes mais provável que os homens bebem mais que as mulheres. Em termos de uso abusivo, os homens (2\%) com idade de 70 a 74 anos bebiam mais que cinco doses de bebidas alcoólicas por semana, enquanto, para as mulheres $(1 \%)$ com mais de 70 anos, este consumo ficou em torno de 3,5 doses de bebidas alcoólicas por semana. ${ }^{5}$

0 baixo nível de escolaridade foi evidenciado em $91 \%$ dos clientes que eram analfabetos ou tinham o ensino fundamental. A literatura evidencia que o baixo nível de escolaridade é considerado um fator de risco potencial para o abuso e dependência de qualquer substância psicoativa. ${ }^{5-6,15}$ Muitas vezes, esses clientes não entendem o uso de diversas substâncias psicoativas que agem como droga causadora de graves consequências à sua saúde, principalmente as drogas lícitas e as medicações prescritas com potenciais de risco para 0 abuso.

A droga lícita de maior uso identificada no presente estudo foi o álcool. Embora a maioria dos estudos internacionais ${ }^{2-8}$ aborde o uso do álcool, como um fenômeno complexo, não muito bem esclarecido, caracterizado como uma epidemia invisível encoberta pelos índices que são subestimados e mal diagnosticados. Um fator crítico evidenciado foi o fato de que os idosos provavelmente desenvolvem muitos problemas de saúde mesmo com níveis relativamente baixos de uso de bebidas alcoólicas. ${ }^{2-9}$ Apesar de os estudos ainda apresentarem dados controversos a respeito das quantidades limites recomendados para o uso do álcool em idosos, recomenda-se que os idosos não devem consumir mais que uma dose por dia de bebida alcoólica (10 a 12 gramas de álcool, por exemplo, equivale a uma lata de cerveja $350 \mathrm{ml}$ ), sete doses por semana e não mais que duas doses em uma única ocasião. ${ }^{5}$

Um estudo demonstrou que, entre os idosos, 2,3\% necessitavam de tratamento para 0 uso de substâncias psicoativas e 85,8\% eram dependentes de álcool ou consumiam em níveis abusivos. ${ }^{16}$

No presente estudo, as medicações de abuso constituíram o segundo maior grupo de drogas usadas pelos idosos do CAPS-ad. E a literatura considera que esse grupo de drogas está dentre os problemas mais significantes entre os idosos, seja no âmbito do abuso ou da dependência de medicamentos prescritos. ${ }^{2-11}$ No Brasil, parece haver uma cultura de automedicação em que para todo e qualquer problema sempre existe um "remedinho" mesmo sem indicação médica, principalmente entre as pessoas mais idosas ("mais experientes").

Além disso, em um estudo de revisão sobre o uso de substâncias psicoativas em idosos, os autores levantaram que um a cada quatro idosos fazia uso de uma medicação com potencial risco de abuso, fato que tem sido um problema muito frequente nessa faixa etária. ${ }^{15}$ As drogas prescritas mais comuns incluem os sedativos, os hipnóticos e os tranquilizantes/ analgésicos, e o uso dessas substâncias são potenciais de risco para o desenvolvimento do abuso e a dependência.

Nesse sentido, a literatura direciona uma atenção especial aos benzodiazepínicos que são as drogas usadas principalmente indicadas no tratamento da ansiedade e depressão, que são comuns em idosos, ${ }^{6,15}$ e evidencia que os riscos de tomar uma medicação prescrita aumentam com 0 avançar da idade.

Os idosos perfazem o maior grupo de uso de drogas legais, contando com mais de $25 \%$ de todas as prescrições. ${ }^{15}$ Os autores apontam que uma particularidade do uso dos sedativo-hipnóticos é que os idosos tendem a tomá-los por um período prolongado.

Investigando as prescrições de 100 idosos que faziam uso de drogas admitidos em um programa de tratamento para dependência de substâncias psicoativas, 35\% apresentaram critérios diagnósticos para dependência da substância (medicação prescrita) em pacientes com mais de 60 anos, com alta correlação entre as mulheres. ${ }^{17}$

Além disso, os idosos também tendem a usar múltiplas drogas, e esse grupo da população consome duas vezes mais medicações quando comparado aos jovens. ${ }^{2}$

Em relação ao uso do tabaco, pouco identificado entre os idosos da presente pesquisa, pode-se pensar que a busca do tratamento não foi espećfica para a dependência da nicotina. Ao contrário de outras drogas, o cigarro foi por muito tempo muito usado e aceito socialmente; apenas com as atuais mudanças nas políticas brasileiras, que restringiram o uso de tabaco em locais públicos, esta tolerância parece estar diminuindo.

Deste modo, nos serviços de saúde, o tabaco nem sempre tem sido avaliado como uma droga de abuso, o que dificulta a identificação do problema. Conforme apresentado na Tabela 2, apenas três idosos buscaram assistência especíica para a dependência da nicotina. Dados empíricos do local de estudo apontam uma prevalência de uso do tabaco entre os usuários do serviço em torno de $70 \%$, e a dependência desta substância nem sempre foco de atenção ou de busca de cuidado e assistência. Estudos internacionais indicaram que $19 \%$ dos homens e $23 \%$ das mulheres idosas (idade acima de 65 anos) fumavam e que $10 \%$ dos homens e $9 \%$ das mulheres (idade acima de 75 anos) eram fumantes atuais. ${ }^{3,18}$

Nos grupos de idosos, a nicotina geralmente tem sido muito pouco avaliada e conta com maior número de incapacidade e mortalidade quando comparada com outras substâncias psicoativas, em função do uso prolongado e de forma pesada. Seu uso se torna preocupante, uma vez que muitos idosos já possuem algum tipo de doença crônica e o hábito de fumar leva à deterioração da saúde. . $^{3-11} 0$ uso do 
tabaco está diretamente relacionado a 25 doenças que ameaçam a vida, e o ato de fumar foi considerado dentre os maiores fatores de risco em oito das 16 causas de morte em pessoas com idade acima dos 60 anos. $^{18}$

Há de considerar que as complicações do fumar ocorrem mais tardiamente ao período de uso e podem, entretanto, manifestar-se na pessoa fumante por muitos anos sem efeitos aparentes. A cessação do fumar tem sido benéfica em todas as idades, com efeitos benéficos imediatos e ao longo do tempo, para as pessoas que possuem ou não doenças relacionadas ao consumo do cigarro. Quase 75\% dos potenciais anos de vida perdidos em função do fumar ocorrem entre as pessoas com idade maior que 60 anos. $^{3}$

No presente estudo, as drogas ilícitas de maior uso foram a maconha, a cocaína e o crack. Porém, evidencia-se $\mathrm{e}^{2,5,15}$ que o uso dessas drogas geralmente não é muito comum em idosos. Apesar das limitações, a literatura sugere cada vez mais que o abuso e a dependência das substâncias ilícitas estão se tornando comuns em idosos que buscaram tratamento especializado. ${ }^{15}$ Embora ainda seja raro, uma vez que o uso de drogas ilícitas atinge menos que $1 \%$ dos idosos, estudos indicaram um aumento considerável deste uso nas próximas décadas, potencializado pelos adultos que estão envelhecendo, ${ }^{1,15}$ lembrando ainda que as taxas de uso de drogas ilícitas podem estar subestimadas e há possibilidade de aumentarem com o envelhecimento da população, uma vez que é elevada entre os jovens. ${ }^{2}$

Ainda a respeito do uso de drogas ilícitas (maconha, cocaína e crack) em idosos, tem sido muito pouco documentado, e essa classe de drogas é limitada a pequenos grupos de idosos que já usavam drogas há muito tempo e estavam envolvidos com a criminalidade. ${ }^{15}$ Este uso foi mais identificado em homens idosos que apresentam maiores probabilidades de abuso de álcool e drogas ilícitas e em mulheres idosas com altas taxas de abuso de sedativos e hipnóticos. ${ }^{19}$

Outro dado importante é que, em serviços especializados, os índices de drogas ilícitas atingem $26 \%$ em pacientes em tratamento para comorbidades.

Estudos realizados em serviços especializados identificaram índices de $26 \%$ de uso drogas ilícitas em pacientes em tratamento para comorbidades. ${ }^{20}$

\section{CONCLUSÃO}

O uso de substância psicoativa em idosos tem sido um tema importante para a Saúde Pública e necessita de especial atenção, por ser complexo e ainda pouco explorado. 0 número de idosos atendidos em serviços especializados para o tratamento do uso de substâncias psicoativas é muito baixo, 0 que leva a repensar a prática assistencial que tem sido oferecida pelos profissionais de saúde.

Embora evidenciado um índice baixo de idosos que usavam substâncias psicoativas, este dado pode estar limitado por falta de informações mais específicas nessa população. Notadamente, a carência de estudos nesta temática, principalmente no Brasil, suscita a necessidade de mais estudos com vistas a elucidar o perfil desta clientela e o progresso da exposição ao uso problemático, bem como documentar fatores de risco e proteção associados ao uso, abuso e dependência das substâncias psicoativas, de forma que possam trazer entendimentos necessários para o desenvolvimento de trabalhos assistenciais, educacionais e preventivos para esses clientes, bem como o desenvolvimento de instrumentos que ajudem os profissionais de saúde a identificar e intervir precocemente tais riscos.

A literatura apresenta com maior ênfase o uso abusivo do álcool em idosos, mas as drogas que tem preocupado muito os especialistas são os medicamentos prescritos, pois esses também podem se tornar de abuso mediante o uso prolongado nessa faixa etária, drogas a princípio usadas por indicações terapêuticas.

Nesse sentido, mais estudos, treinamentos e conscientização por parte dos profissionais de saúde são fundamentais para melhoria da identificação e intervenções eficazes. Recomenda-se que haja a manutenção de boas relações entre os serviços de saúde (atenção primária) que são essenciais para a promoção da continuidade de cuidados e, de particular importância, a articulação entre os serviços de geriatria e os serviços especializados no tratamento do uso de substâncias psicoativas.

Todos os profissionais de saúde envolvidos na assistência de saúde dos idosos, tanto da comunidade como na assistência domiciliar, devem estar conscientes dos problemas potenciais que envolvem o uso de substâncias psicoativas (lícitas e ilícitas) em idosos.

Estudos dessa natureza são importantes para preencher a lacuna existente nesse grupo da população que está em evidente crescimento, assim como do uso das substâncias psicoativas, para que práticas mais efetivas possam ser planejadas e aplicadas no âmbito da prevenção e do tratamento

\section{REFERÊNCIAS}

1. Instituto Brasileiro de Geografia e Estatística- IBGE. Indicadores sociodemográficos e de saúde no Brasil; 2009.

2. Crome IB, Day E. Substance misuse and dependence: older people deserve better services. Rev Clin Gerontol. 1999; 9: 327-42

3. Philips P, Katz A. Substance misuse in older adults: an emerging policy priority. Res Nurs. 2001; 6(6): 898-905. 
4. Levin SM, Kruger J. Substance abuse among older adults: a guide for social services providers. Rockville, MD: U.S. Department of Health and Human Services, Substance Abuse and Mental Health Services Administration. Center for Substance Abuse Treatment; 2000.

5. Dar K. Alcohol use disorders in elderly people: fact or fiction? Adv Psychiatr Treat. 2006; 12: 173-81.

6. Dufour M, Fuller RK. Alcohol in the elderly. Annu Rev Med 46:123-132, 1995.

7. O'Connell H, Chin AV, Cunningham C, Lawlor B. Alcohol use disorders in elderly people redefining an age old problem in old age. BMJ: british medical journal 2003; 327: 664-67.

8. Menninger J. Assessment and treatment of alcoholism and substance related disorders in the elderly. Bull Menninger Clin. 2002; 66(2), 166-83.

9. Rigler SK. Alcoholism in the elderly. Am Fam Physician. 2000; 61 (6): 171016.

10. Bell AJ, Talbot-Stern JK, Hennessy A. Characteristics and outcomes of older patients presenting to the emergency department after a fall: a retrospective analysis. Med J Aust. 2000; 173:179.

11. McLnnes E, Powell J. Drug and alcohol referrals; are elderly substance abuse diagnoses and referrals being made. BMJ: british medical journal 1994; 308: 444-46

12. National Household Survey on Drug Abuse, Summary of National Findings. Rockville, MD: Office of Applied Studies; 2002. v.1.

13. Boyle AR, Davis H. Early screening and assessment of alcohol and substance abuse in the elderly: clinical implications. J Addict Nurs. 2006; 17: 95-103.

14. Curtis JR, Geller G, Stokes EG, et al: Characteristics, diagnosis and treatment of alcoholism in elderly patients. J Am Geriatr Soc. 1989; 37: 310-16.

15. Simoni-Wastila L, Yang HK. Psychoactive drug abuse in older adults. Am J Geriatr Pharmacother. 2006; 4(4): 380-94.

16. Gfroerer J, Penne M, Pemberton M, Folsom R. Substance abuse treatment need among older adults in 2020: the impact of the aging babyboom cohort. Drug Alcohol Depend. 2003; 69(2): 127-35.

17. Finlayson RE, Davis $\sqcup J$ Jr. Prescription drug dependence in the elderly population: demographic and clinical features of 100 inpatients. Mayo Clin Proc. 1994, 69(12): 1215-17.

18. Department of Health. Smoking kills: a white paper on tobacco. London: Stationary Office; 1998. CM 4177.

19. Atkinson RM, Ganzini L, Bernstein MJ. Alcohol and substance-use disorders in the elderly. In: Birren JE, Sloane RB, Cohen GD, editores. Handbook of mental health and aging, 2 nd ed. San Diego, CA: Academic Press; 1992.

20. Prigerson HG; Desai RA; Rosenheck RA. Older adult patients with both psychiatric and substance abuse disorders: prevalence and health service use. Psychiatric Quaterly. 2001; 72: 1-18. 